\title{
Predicción del comportamiento hidrodinámico en el escalado de un reactor de tanque agitado para procesos aerobios, mediante CFD
}

\author{
CFD prediction of hydrodynamic behavior in the scale up \\ of a stirred tank reactor for aerobic processes \\ Ricardo Gelves $^{1} \quad$ Aldo Benavides $^{2} \quad$ Juan Carlos Quintero $^{3}$ \\ Recibido 6 de diciembre de 2011, aceptado 22 de abril de 2013 \\ Received: December 6, $2011 \quad$ Accepted: April 22, 2013
}

\begin{abstract}
RESUMEN
En el presente trabajo se analiza mediante simulaciones en CFD (Dinámica de Fluidos Computacional) el comportamiento de la hidrodinámica líquido-gaseosa en el escalado de un biorreactor, aplicando criterios empíricos comúnmente utilizados en procesos biotecnológicos (Número de Reynolds Re y Potencia por unidad de volumen P/V) en reactores de tanque agitado de 0,050 y $0,500 \mathrm{~m}^{3}$. Los efectos de la turbulencia, flujo rotativo, ruptura y coalescencia de burbujas son simulados mediante los modelos k- $\varepsilon$, MRF (Multiple Reference Frame) y PBM (Population Balance Model). El criterio de escalado P/V presenta mejores similitudes hidrodinámicas que el criterio de escalado Re respecto del reactor de $0,050 \mathrm{~m}^{3}$; sin embargo, el alto consumo de potencia y el posible daño microbiano generado por la turbulencia en cultivos de células animales pueden ser desventajas en prototipos a gran escala.

Palabras clave: Biorreactor, dinámica de fluidos computacional (CFD), marco de referencia múltiple (MRF), método de balance poblacional (PBM), escalado.

ABSTRACT

In this paper the effects of some empirical scale up criteria (Re y P/V) on hydrodynamics are analyzed by using CFD simulations (Computational Fluid Dynamics) in 0.050 and $0.500 \mathrm{~m}^{3}$ stirred tank bioreactors. The effects of turbulence, rotating flow, bubbles breakage and coalescence were simulated by using the $k$-E, MRF (Multiple Reference Frame) and PBM (Population Balance Model), respectively. The P/V scale up criteria showed better similar hydrodynamics than Re scale up criteria with respect to the $0.050 \mathrm{~m}^{3}$ reactor. However, the high power consumption and cellular damage due to turbulence in animal cell cultures could be disadvantages in large scale prototypes of bioreactors.
\end{abstract}

Keywords: Bioreactor, computational fluid dynamics $(C F D)$, multiple reference frame (MRF), population balance model (PBM), scale up.

\section{INTRODUCCIÓN}

El éxito de muchos procesos biotecnológicos a escala industrial depende de una agitación y aireación efectiva de los fluidos presentes. La hidrodinámica desarrollada en un biorreactor promueve la transferencia de masa y calor entre las fases y la distribución uniforme de los nutrientes durante un proceso fermentativo. Durante el cambio de escala surgen problemas asociados con la hidrodinámica de biorreactores que consecuentemente reducen, en algunos casos, la productividad de un bioproceso,

1 Grupo de Biotecnología. Sede de Investigación Universitaria-SIU. Universidad de Antioquia. Carrera 53 No 61-30. Medellín, Colombia. E-mail: egrgz832@udea.edu.co

2 Grupo de Bioinstrumentación e Ingeniería Clínica. Universidad de Antioquia. Calle 67 N. 53-108. Medellín, Colombia. E-mail: aldogbmoran@gmail.com

3 Grupo de Bioprocesos. Universidad de Antioquia. Calle 67 N. 53-108. Medellín, Colombia. E-mail: jcquinte@udea.edu.co 
respecto de escalas de laboratorio [1-3]. El alejamiento de las condiciones de mezcla completa con el incremento de la escala, genera gradientes que conducen a un distanciamiento de las condiciones óptimas encontradas en condiciones de laboratorio. Gradientes de gases, esfuerzos cortantes, entre otros, en fluidos multifásicos que comúnmente son utilizados en procesos biotecnológicos, son causados por una deficiente mezcla generada por la adopción de métodos empíricos como estrategias de escalado. Todos los criterios empíricos de escalado como Re (Número de Reynolds constante) y P/V (Potencia por unidad de volumen constante), entre otros, se basan en aproximaciones ideales para mantener un parámetro de operación constante en ambas escalas, dejando de lado la hidrodinámica y los fenómenos de transporte que gobiernan el proceso de escalado. Conocer el comportamiento hidrodinámico durante la operación de biorreactores a diferentes escalas de operación permite identificar el grado de alejamiento de la mezcla perfecta que viene asociado cuando se emplean los diferentes criterios empíricos en el escalado. La dinámica de fluidos computacional (CFD del inglés Computational Fluid Dynamics) es una muy útil herramienta para analizar en detalle el fenómeno de la turbulencia en biorreactores de tanque agitado, los cuales son comúnmente usados en procesos de fermentaciones microbianas. Para la simulación en CFD del escalado de un proceso aerobio es importante considerar fenómenos como la ruptura y coalescencia de burbujas a partir de modelos de balance poblacional, ya que en el biorreactor estos fenómenos desencadenan la formación de zonas poco oxigenadas y gradientes de gases que pueden afectar la productividad durante el escalado debido a que: i) El comportamiento de las burbujas tiene una influencia significativa en la transición del régimen de flujo porque el fenómeno más importante que causa esta transición desde un régimen homogéneo a un régimen heterogéneo es la ocurrencia de grandes burbujas, ii) El tamaño de las burbujas tiene una amplia distribución en un régimen heterogéneo que comúnmente se presenta en un proceso de cambio de escala [4], iii) Las velocidades radiales, axiales y tangenciales de las burbujas y la disipación de la energía cinética turbulenta influyen significativamente en los fenómenos de ruptura y coalescencia que como consecuencia definen los perfiles de gradientes de aire en un biorreactor. En los últimos años ha habido un rápido avance en el modelado a través de CFD (Dinámica de Fluidos Computacional) de sistemas de agitación de reactores, incluyendo desde modelos de rotación de fluidos en una fase [5-9] hasta la utilización de modelos de balance poblacional en sistemas de agitación líquido-gaseosa [10-27]. Sin embargo, no existen en la actualidad investigaciones que analicen mediante predicciones en CFD (Dinámica de Fluidos Computacional), los efectos que podrían generarse en fenómenos como la ruptura y coalescencia de burbujas, como consecuencia de comportamientos no ideales en la hidrodinámica generados por la aplicación de criterios empíricos comúnmente utilizados durante la etapa de escalado de biorreactores de tanque agitado. Por tal motivo en el presente trabajo se pretende identificar comportamientos no ideales generados durante estudios de escalado entre 0,050 y $0,500 \mathrm{~m}^{3}$ empleando dos criterios empíricos de escalado utilizados comúnmente en bioprocesos. Para esto se realizaron simulaciones con el software de CFD (Dinámica de Fluidos Computacional) ANSYSFLUENT v13 que permite obtener información sobre parámetros hidrodinámicos como perfiles de dispersión de gases, turbulencia, distribución de tamaños de burbujas, etc. Esta estrategia de predicción de problemas que se pueden generar durante la etapa de escalado de un bioproceso puede evitar la necesidad de construir prototipos costosos e ineficientes, lo que traería como ventaja un ahorro en tiempo de experimentación y presupuesto para una empresa del sector biotecnológico.

\section{MATERIALES Y MÉTODOS}

\section{Características físicas del reactor de $0,050 \mathrm{~m}^{3}$}

Para la etapa inicial (volumen de referencia) se utilizó un biorreactor de tanque agitado New Brunswick BioFlo 5000 a escala piloto $\left(0,080 \mathrm{~m}^{3}\right.$ de volumen total) con $0,050 \mathrm{~m}^{3}$ de agua como volumen de trabajo (temperatura de $293 \mathrm{~K}$ y presión de $101325 \mathrm{~Pa}$ ). El tanque está caracterizado por las siguientes dimensiones: diámetro $\mathrm{D}_{\mathrm{T}}: 0,380 \mathrm{~m}$, altura del líquido $\mathrm{H}_{\mathrm{L}}: 0,380 \mathrm{~m}$, equipado con cuatro deflectores espaciados en un ángulo de $90^{\circ}$ con una anchura $\mathrm{W}_{\mathrm{b}}$ : 0,1 $\mathrm{D}_{\mathrm{T}}$ instalados a $0,010 \mathrm{~m}$ de la pared del tanque. El equipo cuenta con un impulsor tipo turbina Rushton (disco con seis paletas rectas y perpendiculares) con un diámetro $\mathrm{D}_{\mathrm{i}}: 0,1265$ $\left(\mathrm{D}_{\mathrm{i}}: 0,3 \mathrm{D}_{\mathrm{T}}\right)$, ancho de la paleta $\mathrm{W}_{\mathrm{i}}: 0,030 \mathrm{~m}\left(\mathrm{~W}_{\mathrm{i}}\right.$ : $\left.0,25 \mathrm{D}_{\mathrm{i}}\right)$, altura de la paleta $\mathrm{W}_{\mathrm{L}}: 0,040 \mathrm{~m}\left(\mathrm{~W}_{\mathrm{L}}: 0,33\right.$ $\mathrm{D}_{\mathrm{i}}$ :). El aire es suministrado a través de un difusor 
de $0,125 \mathrm{~m}$ de diámetro localizado debajo de la turbina a una altura de 0,09 m. Para la etapa de escalado virtual simulada en CFD se dimensionó un biorreactor de $0,500 \mathrm{~m}^{3}\left(\mathrm{D}_{\mathrm{T}}: 0,75 \mathrm{~m} \mathrm{y}_{\mathrm{L}}\right.$ : $1,1 \mathrm{~m}$ )con una similitud geométrica respecto de biorreactor de $0,050 \mathrm{~m}^{3}$. Las propiedades utilizadas para la fase primaria (agua) fueron: $\rho_{\mathrm{L}}: 998,2 \mathrm{kgm}^{-3}$; $\mu_{\mathrm{L}}: 0,001 \mathrm{kgm}^{-1} \mathrm{~s}^{-1} \cdot \sigma: 0,073 \mathrm{Nm}^{-1}$. Las Propiedades de la fase secundaria (aire) fueron: $\rho_{\mathrm{G}}: 1,225 \mathrm{kgm}^{-3}$, $\mu_{\mathrm{G}}=1,78910^{-5} \mathrm{kgm}^{-1} \mathrm{~s}^{-1}[28]$.

\section{Criterios empíricos de escalado}

Las condiciones de operación para el proceso de escalado a $0,500 \mathrm{~m}^{3}$ se calcularon con base en los criterios Re y P/V teniendo en cuenta la velocidad de agitación $N_{i}$ del biorreactor de $0,050 \mathrm{~m}^{3}$ (400 rpm) y manteniendo constante el flujo de aire a $1 \mathrm{vvm}$ en ambas escalas.

Criterio número de Reynolds constante Re. El escalado con base en el número de Reynolds asume que la magnitud relativa de los esfuerzos inerciales respecto de los esfuerzos viscosos se mantiene constante en ambas escalas. Si los esfuerzos inerciales dominan respecto de los esfuerzos viscosos se dice que el flujo no puede ser laminar. A la pérdida de laminaridad se le denomina turbulencia y esta es muy importante para la transferencia de masa y dispersión de gases. En esta investigación se utilizó el criterio Número de Reynolds constante, ya que por su carácter teórico y empírico define una supuesta semejanza en el patrón de flujo hidrodinámico, lo que significa que adoptando este criterio se trataría de mantener el mismo grado de turbulencia, en ambas escalas [29]. Con el incremento de escala, la velocidad de agitación requerida para mantener Re constante se reduce significativamente y el grado de heterogeneidad puede aumentar, por lo que se generarían comportamientos no ideales que podrían ser identificados a través de Dinámica de Fluidos Computacional.

Criterio potencia por unidad de volumen constante $\mathrm{P} / \mathrm{V}$. El criterio de escalado P/V se basa en mantener constante el consumo de potencia por unidad de volumen de un proceso líquido-gaseoso debido a la agitación [29]. P/V requiere de un mayor grado de agitación respecto de la velocidad requerida para mantener el criterio Re constante. Es por tal motivo que su desventaja radica en el alto consumo de potencia, ya que la velocidad en la punta del agitador se incrementa con la escala y, por lo tanto, el diseño de un motor de agitación a gran escala resulta poco práctico en los bioprocesos. Como consecuencia del incremento en la velocidad en la punta del agitador, el esfuerzo cortante puede ocasionar daños en la morfología de células microbianas. Sin embargo, una agitación y aireación efectiva de los fluidos presentes en un biorreactor promueven la dispersión de gases y tamaño de burbujas para la transferencia de masa. Lo anterior soporta las grandes diferencias significativas en la hidrodinámica líquido-gaseosa que podrían presentarse utilizando los criterios $\mathrm{P} / \mathrm{V}$ y Re. Por esta razón en esta investigación se han incluido estos criterios de escalado, ya que se busca predecir a través de CFD tendencias hidrodinámicas opuestas generadas según el criterio de escalado empleado (P/V y Re).

\section{Modelo de CFD}

\section{Ecuaciones de Navier-Stokes}

La fase gaseosa y la fase líquida son tratadas como series continuas de interpenetración y las ecuaciones de conservación de masa y momentum se resuelven para cada fase. Debido a que el volumen de una fase no puede ser ocupado por las otras fases se utiliza el concepto de fracción de volumen que son funciones continuas en el espacio y el tiempo. Las ecuaciones de conservación para cada fase se derivan para obtener un sistema de ecuaciones, que tienen estructura similar para todas las fases [17-22].

El modelo euleriano es el más complejo de los modelos multifásicos en ANSYS FLUENT v13. Soluciona un sistema de n-ecuaciones de momentum y continuidad para cada fase presente. El acoplamiento se alcanza a través de la presión y de los coeficientes de intercambio interfacial. La ecuación de conservación de masa para cada fase se muestra a continuación:

$$
\frac{\partial}{\partial t}\left(\rho_{i} \alpha_{i}\right)+\nabla\left(\alpha_{i} \rho_{i} \vec{U}_{i}\right)
$$

Donde $\rho_{i} \alpha_{i}$ y $\vec{U}_{i}$ representan la densidad, la fracción de volumen y la velocidad de la fase i (G o L). Se asume que la fase líquida $\mathrm{L}$ y la fase gaseosa $\mathrm{G}$ comparten el espacio en proporción a su volumen, de manera que el total de las fracciones suman un valor igual una unidad. 


$$
\alpha_{G}+\alpha_{L}=1,0
$$

La ecuación de momentum para la fase i se describe a continuación:

$$
\begin{aligned}
& \frac{\partial}{\partial t}\left(\rho_{i} \alpha_{i} \vec{U}_{i}\right)+\nabla \cdot\left(\alpha_{i} \rho_{i} \vec{U}_{i} \vec{U}_{i}\right)=\alpha_{i} \nabla p \\
& +\nabla \cdot \overline{\bar{\tau}}_{\text {effi }}+\vec{R}_{i}+\vec{F}_{i}+\alpha_{i} \rho_{i} \vec{g}
\end{aligned}
$$

$p$ es la presión compartida por ambas fases, $\vec{g}$ es la gravedad y $\vec{R}_{i}$ representa el intercambio de momentum interfaz. El término $\vec{F}_{i}$ representa las fuerzas de Coriolis y centrífuga expresada en el modelo para flujos rotativos MRF y se representa como:

$$
\vec{F}_{i}=-2 \alpha_{i} \rho_{i} \vec{N} \times \vec{U}_{i}-\alpha_{i} \rho_{i} \vec{N} \times(\vec{N} \times \vec{r})
$$

$\vec{N}$ es la velocidad angular, $\vec{r}$ es la posición vectorial. El tensor de esfuerzos de Reynolds $\overline{\bar{\tau}}_{\text {effi }}$ se relaciona con los gradientes medios de velocidad mediante la hipótesis de Boussinesq [17]:

$$
\begin{aligned}
& \overline{\bar{\tau}}_{\text {effi }}=\alpha_{i}\left(\mu_{\text {lam }, i}+\mu_{t, i}\right)\left(\nabla \vec{U}_{i}+\nabla \vec{U}_{i}^{T}\right) \\
& -\frac{2}{3} \alpha_{i}\left(\rho_{i} k_{i}+\left(\mu_{\text {lam }, i}+\mu_{t, i}\right) \nabla \cdot \vec{U}_{i}\right) \overline{\bar{I}}
\end{aligned}
$$

$\mu_{\text {lam }, i}$ es la viscosidad molecular de la fase i, $\overline{\bar{I}}$ es el tensor de deformación, $\mu_{t, i}$ es la viscosidad turbulenta de la fase i. $k_{i}$ es la energía cinética turbulenta.

\section{Intercambio de momentum interfacial}

La fuerza más importante de intercambio entre las fases es la fuerza de arrastre $R_{G}$ que actúa sobre las burbujas que funciona como una fuerza de cerradura para ambas fases en las ecuaciones de momentum. Esta fuerza depende de la fricción, de la presión, de la cohesión y de otros efectos hidrodinámicos [30].

$$
R_{L}=-R_{G}=K\left(\vec{U}_{G}-\vec{U}_{L}\right)
$$

$K$ es el coeficiente de intercambio de las fases líquida y gaseosa y se determina con la ecuación:

$$
K=\frac{3}{4} \rho_{L} \alpha_{L} \alpha_{G} \frac{C_{D}}{d}\left|\vec{U}_{G}-\vec{U}_{L}\right|
$$

$d$ es el diámetro de burbuja y $C_{D}$ es el coeficiente de arrastre que se define como una función de número de Reynolds $R e_{p}$ :

$$
\operatorname{Re}_{p}=\frac{\rho_{L}\left|\vec{U}_{G}-\vec{U}_{L}\right| d}{\mu_{L}}
$$

Para el cálculo del coeficiente de arrastre se utiliza la correlación estándar [31]:

$$
C_{D}=\left\{\begin{array}{ll}
\frac{24\left(1+0,15 R e_{p}^{0,687}\right)}{R e_{p}}, & R e_{p d} \leq 1000 \\
0,44, & R e_{p d}>1000
\end{array}\right\}
$$

\section{Ecuaciones para el modelo de turbulencia}

El modelo de turbulencia $k-\varepsilon$ disperso es el modelo multifásico de turbulencia estándar. Representa la extensión del modelo monofásico $k-\varepsilon$ y es aplicable cuando las concentraciones de la fase secundaria son diluidas. Las ecuaciones de $k$ y $\varepsilon$ que describen este modelo son como sigue:

$$
\begin{gathered}
\frac{\partial}{\partial t}\left(\rho_{L} \alpha_{L} k_{L}\right)+\nabla \cdot\left(\rho_{L} \alpha_{L} \vec{U}_{L} k_{L}\right) \\
=\nabla \cdot\left(\alpha_{L} \frac{\mu_{t, L} \nabla k_{L}}{\sigma k}\right)+\alpha_{L} G_{k, L}-\alpha_{L} \rho_{L} \varepsilon_{L} \\
+\alpha_{L} \rho_{L} \Pi_{K, L}
\end{gathered}
$$

$\frac{\partial}{\partial t}\left(\rho_{L} \alpha_{L} k_{L} \varepsilon_{L}\right)+\nabla \cdot\left(\rho_{L} \alpha_{L} \vec{U}_{L} \varepsilon_{L}\right)$

$$
\begin{aligned}
=\nabla \cdot\left(\alpha_{L} \frac{\mu_{t, L} \nabla \varepsilon_{L}}{\sigma \varepsilon}\right) & +\alpha_{L} \frac{\varepsilon_{L}}{k_{L}}\left(C_{1 \varepsilon} G_{k, L}-C_{2 \varepsilon} \rho_{L} \varepsilon_{L}\right) \\
& +\alpha_{L} \rho_{L} \Pi_{\varepsilon, L}
\end{aligned}
$$

En estas ecuaciones, $G_{k, l}$ representa la generación de energía cinética turbulenta $k_{L}$ de la fase líquida debido a los gradientes de la velocidad media, $\varepsilon_{L}$ es la disipación de la energía cinética turbulenta. $\Pi_{K, L}$ y $\Pi_{\varepsilon, L}$ representan la influencia de la fase dispersa en la fase continua y son modeladas con las ecuaciones de Elgobashi y Rizk [32].

La viscosidad turbulenta $\mu_{t, L}$ es calculada de:

$$
\mu_{t, L}=\rho_{L} C_{\mu} \frac{k_{L}^{2}}{\varepsilon_{L}}
$$


Los valores de las constantes utilizados en este experimento fueron $C_{1 \varepsilon}: 1,44 C_{2 \varepsilon}: 1,92 C_{\mu}: 0,09$ $\sigma_{k}: 1,00$ y $\sigma_{\varepsilon}: 1,30 . \sigma_{k}$ y $\sigma_{\varepsilon}$ representan el número de Prandtl turbulento para $k$ y $\varepsilon$, respectivamente.

\section{Método de balance poblacional}

En este trabajo se utilizó el método discreto [3335] para solucionar las ecuaciones de balance poblacional por su robustez numérica, ya que la población de burbujas es discretizada en un número finito de intervalos de diámetros. Las ecuaciones de balance poblacional para las diferentes clases de burbujas en el reactor se pueden escribir como [27, 36-37]:

$$
\begin{aligned}
& \frac{\partial}{\partial t}\left(\rho_{G} n_{i}\right)+\nabla \cdot\left(\rho_{G} \vec{U}_{G} n_{i}\right) \\
& =\rho_{G}\left(B_{i_{C}}-D_{i_{C}}+B_{i_{B}}-D_{i_{B}}\right)
\end{aligned}
$$

Donde $n_{i}$ es el número de clases de burbujas $i, B_{i_{C}}$ y $B_{i_{B}}$ son las velocidades de nacimiento debidas a la coalescencia y rotura, respectivamente, $D_{i_{C}}$ y $D_{i_{B}}$ son las tasas de mortalidad.

Los términos de rotura y coalescencia son:

$$
\begin{gathered}
B_{i_{C}}=\frac{1}{2} \int_{0}^{v} a\left(v-v^{\prime}, v\right) n(v \\
\left.-v^{\prime}, t\right) n\left(v^{\prime}, t\right) d v^{\prime} \\
D_{i_{C}}=n(v) \int_{0}^{\infty} a\left(v, v^{\prime}\right) n\left(v^{\prime}, t\right) n\left(v^{\prime}, t\right) d v^{\prime} \\
B_{i_{B}}=\int_{\Omega v} p g\left(v^{\prime}\right) \beta\left(v \mid v^{\prime}\right) n\left(v^{\prime}, t\right) d v^{\prime} \\
D_{i_{B}}=g(v) n(v, t)
\end{gathered}
$$

$a\left(v, v^{\prime}\right)$ es la tasa de coalescencia entre las burbujas de tamaño $v$ y $v^{\prime} ; g(v)$ es la tasa de ruptura de burbujas de tamaño $v ; g\left(v^{\prime}\right)$ es la frecuencia de ruptura de burbujas $v^{\prime}$ y $\beta\left(v \mid v^{\prime}\right)$ es la probabilidad de la función densidad de las burbujas rotas desde el volumen $v^{\prime}$ a una burbuja de volumen $v ; p$ es el número de burbujas formadas por cada rompimiento; $n$ es el número de clases de burbujas y $\Omega_{v}$ es el volumen de una burbuja $v$. La ruptura de burbujas es analizada en términos de la interacción de las burbujas con los remolinos turbulentos. Estos remolinos turbulentos incrementan la energía de superficie de las burbujas hasta causar la deformación. La ruptura ocurre si el incremento en la energía de superficie alcanza un valor crítico. La tasa de ruptura se define como [6]:

$$
\begin{gathered}
g\left(v^{\prime}\right) \beta\left(v \mid v^{\prime}\right) \\
=k \int_{\xi \min }^{1} \frac{(1+\xi)^{2}}{\xi^{11 / 3}} \exp \left(-b \xi^{-11 / 3}\right) d \xi \\
k=0.9238 \varepsilon^{1 / 3} d^{-2 / 3 \alpha} \\
b=12\left(f^{2 / 3}+(1-f)^{2 / 3}\right. \\
-1) \sigma \rho^{-1} \varepsilon^{-2 / 3} d^{-5 / 3}
\end{gathered}
$$

Donde $d$ es el diámetro de partícula, $\xi$ es el tamaño adimensional de remolinos, $f$ es la frecuencia de rompimiento, $\sigma$ es la tensión superficial y $\alpha$ es la fracción de partículas. La coalescencia de burbujas se modela considerando la colisión de burbujas debida a la turbulencia, flotabilidad y corte laminar. La tasa de coalescencia se define como el producto entre la frecuencia de colisión $\omega_{a g}\left(v_{i}, v_{j}\right)$ y la probabilidad de coalescencia $P_{a g}\left(v_{i}, v_{j}\right)$ y se define como [6]:

$$
a\left(v, v^{\prime}\right)=\omega_{a g}\left(v_{i}, v_{j}\right) P_{a g}\left(v_{i}, v_{j}\right)
$$

La frecuencia de colisión se define como:

$$
\omega_{a g}\left(v_{i}, v_{j}\right)=\frac{\pi}{4}\left(d_{i}^{2}+d_{j}^{2}\right) n_{i} n_{j} \bar{u}_{i j}
$$

Donde $\bar{u}_{i j}$ es la velocidad característica de colisión de dos partículas con diámetro $d_{i}$ y $d_{j}$ y densidad de burbujas $n_{i}$ y $n_{j}$

$$
\begin{aligned}
& P_{a g}\left(v_{i}, v_{j}\right)= \\
& =\exp \left(-c_{1} \frac{\left[0.75\left(1+x_{i j}^{2}\right)\left(1+x_{i j}^{3}\right)\right]^{1 / 2}}{\left(\rho_{2} / \rho_{1}+0.5\right)^{1 / 2}\left(1+x_{i j}^{3}\right)}\left(\frac{\rho_{l} d_{i}\left(\bar{u}_{i}^{2}+\bar{u}_{j}^{2}\right)^{1 / 2}}{\sigma}\right)^{1 / 2}\right)
\end{aligned}
$$

Donde $c_{1}$ es una constante de primer orden, $x_{i j}=d_{i} / d_{j}$ $\rho_{1}$ y $\rho_{2}$ son las densidades de las fases primaria (agua) y secundaria (aire).

El número de clases de burbujas $i, n_{i}$ se relaciona con la fracción de volumen de la fase gaseosa, de la siguiente manera:

$$
n_{i} v_{i}=\alpha_{i}
$$


La sumatoria de las fracciones en volumen de cada grupo de burbujas es igual a la fracción en volumen de la fase dispersa:

$$
\sum_{i} \alpha_{i}=\alpha_{G}
$$

La fracción en volumen de cada grupo de tamaños se expresa en términos de la fracción total de la fase dispersa:

$$
f_{i}=\frac{\alpha_{i}}{\alpha_{G}}
$$

Por lo tanto, incluyendo la ecuación (26) en la ecuación (13), resulta:

$$
\begin{aligned}
& \frac{\partial}{\partial t}\left(\alpha_{G} \rho_{G} f_{i}\right)+\nabla \cdot\left(\alpha_{G} \rho_{G} \vec{U}_{G} f_{i}\right) \\
& =\rho_{G} v_{i}\left(B_{i_{C}}-D_{i_{C}}+B_{i_{B}}-D_{i_{B}}\right)
\end{aligned}
$$

Para acoplar las ecuaciones de balance poblacional con la hidrodinámica del reactor se utiliza el diámetro de sauter $d_{32}$ como valor de entrada en la ecuación:

$$
d_{32}=\frac{\sum_{i} n_{i} d_{i}^{3}}{\sum_{i} n_{i} d_{i}^{2}}
$$

\section{Especificaciones de los reactores y técnica numérica}

El dominio de solución para esta investigación se presenta en la Figura 1. Se utilizó el software AnsysFluent 13 [38] para generar la geometría y mallado de los reactores. La malla híbrida en 3D es transformada a celdas poliédricas y está constituida por 450,095 celdas para el reactor de $0,05 \mathrm{~m}^{3}$ y 700,000 celdas para el reactor de $0,500 \mathrm{~m}^{3}$. La calidad del mallado se analiza mediante el criterio skewness [38]. En esta investigación todas las celdas presentan un valor skewness inferior a 0,70 , lo que indica que el mallado es aceptable [38]. Se utilizó la técnica de volúmenes finitos [30] para convertir las ecuaciones de Navier-Stokes en ecuaciones algebraicas que pueden ser resueltas numéricamente. Las paredes del tanque, las superficies del agitador y los bafles en los biorreactores se tratan como condiciones de no deslizamiento y funciones de pared estándar.

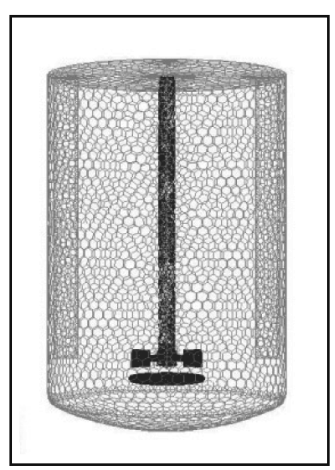

Figura 1. Mallado en 3D del biorreactor de $0,05 \mathrm{~m}^{3}$ con 450,095 celdas.

En la superficie líquida de los biorreactores se adicionó una pequeña zona de aire (Pressure outlet) para evitar que el líquido se escape del tanque [11], por lo tanto, solo se permite que se escape el gas. El flujo de gas en el difusor se define como una condición de entrada (velocity inlet) con una fracción de volumen de gas igual a una unidad.

Para solucionar las ecuaciones diferenciales parciales se utilizó el algoritmo PC SIMPLE que acopla la presión y velocidad. También se utilizó una discretización implícita de primer orden para la integración del término transitorio y un esquema Upwind de segundo orden para los términos espaciales. Se asume que la solución converge cuando los residuales escalados permanecen con valores menores a $10^{-5}$.

\section{Técnicas experimentales y correlaciones utilizadas} Para verificar la validez de las simulaciones se determinó experimentalmente el valor de $k_{L}$ a escala de $0,05 \mathrm{~m}^{3}$ y se comparó con valores obtenidos por CFD. Adicionalmente se utilizaron correlaciones de la bibliografía para verificar las simulaciones a escala de $0,5 \mathrm{~m}^{3}$. Para la determinación experimental de $k_{L} a$, el biorreactor de $0,05 \mathrm{~m}^{3}$ es gasificado con nitrógeno $\left(\mathrm{N}_{2}\right)$ hasta alcanzar una concentración mínima de oxígeno disuelto. En esta etapa se adiciona aire al biorreactor a través del difusor y se toman mediciones temporales de oxígeno disuelto $C_{L}$ hasta que se alcance la saturación. La concentración de oxígeno disuelto se mide utilizando un sensor polarográfico de METTLER TOLEDO. La velocidad de cambio de oxígeno en la fase líquida es determinada usando la siguiente ecuación: 


$$
\frac{d C_{L}}{d t}=k_{L} a\left(C^{*}-C_{L}\right)
$$

Donde $C^{*}$ es la concentración de saturación de oxígeno disuelto. Integrando la ecuación (29) es posible obtener la siguiente ecuación:

$$
\ln \left(\frac{C^{*}-C_{L}}{C^{*}}\right)=k_{L} a . t
$$

Graficando el término izquierdo de la ecuación (30) en función del tiempo se obtiene el valor experimental de $k_{L} a$.

Para calcular la velocidad en la punta del impulsor $N D a$ se utilizó la siguiente correlación:

$$
N D a=\pi N_{i} D_{i}
$$

Para calcular la potencia consumida de una turbina Rushton con aireación, Nagata [43] determinó la siguiente correlación a partir de datos experimentales:

$$
\begin{aligned}
& \log \left(\frac{P_{g}}{P}\right)= \\
& -192\left(\frac{D_{i}}{D_{T}}\right)^{4,38}\left(\frac{D_{i}^{2} N}{v}\right)^{0,115}\left(\frac{D_{i} N^{2}}{9}\right)^{1,96\left(\frac{D_{i}}{D_{T}}\right)}\left(\frac{Q}{N D_{i}^{3}}\right)
\end{aligned}
$$

Donde $P_{g}$ es la potencia consumida con aireación, $P$ potencia consumida sin aireación, $v$ velocidad del gas y $Q$ flujo volumétrico del gas. Las demás variables ya han sido definidas anteriormente.

Se calcularon los valores del torque $M$ del impeler para determinar la potencia consumida mediante CFD, para compararlos con los valores obtenidos por las correlaciones antes mencionadas y verificar la validez de las simulaciones utilizando la siguiente expresión matemática:

$$
P_{g}=2 \pi N_{i} M
$$

La teoría de la penetración de Hibies fue usada para calcular los valores de $k_{L} a$ a partir de CFD:

$$
k_{L}=\frac{2}{\sqrt{\pi}} \sqrt{D_{C_{L}}}\left(\frac{\varepsilon_{L} \rho_{L}}{\mu_{L}}\right)^{\frac{1}{4}}
$$

$$
a=\frac{6 \alpha_{L}}{d_{32}}
$$

$k_{L}$ es el coeficiente de transferencia de la fase líquida, $D_{C_{L}}$ es la difusividad del oxígeno, $a$ es el área interfacial de las burbujas, $d_{32}$ es el diámetro de sauter y $\mu_{L}$ es la viscosidad molecular de la fase líquida.

\section{RESULTADOS Y DISCUSIONES}

El objetivo principal de esta investigación fue predecir el comportamiento hidrodinámico en el escalado de un proceso aerobio utilizando CFD (Dinámica de Fluidos Computacional). Se hizo un énfasis especial en la hidrodinámica y la transferencia de masa líquido-gaseosa. Las condiciones de operación y criterios de escalado simulados en este trabajo se basan en valores comúnmente usados en cultivos de microorganismos aerobios. En la Figura 2 se observan los contornos de distribución de la fase dispersa (aire) en los biorreactores de 0,050 y $0,500 \mathrm{~m}^{3}$ respectivamente, calculados mediante CFD aplicando los criterios de escalado Re y P/V. Usando el criterio de escalado Re en el biorreactor de $0,500 \mathrm{~m}^{3}$ (Figura $2 \mathrm{~b}$ ) se observa poca distribución del gas, especialmente en el fondo del biorreactor, en comparación con el biorreactor de $0,050 \mathrm{~m}^{3}$ (Figura 2 a) debido a las bajas fuerzas centrífugas generadas por la turbina Rushton que no son suficientes para superar el fenómeno de flotabilidad del gas (reflejado en los perfiles de velocidad del aire, Figura 4 b) ocasionando de esta forma ambientes heterogéneos (poca dispersión de aire en el fondo del biorreactor) y gradientes que pueden afectar el crecimiento de un microorganismo aerobio. Teniendo en cuenta la transferencia de masa simulada mediante CFD (Figura 6 b) se observa que en estas zonas del biorreactor, la transferencia de oxígeno es muy pobre. Este fenómeno de flotabilidad es más pronunciado con el criterio de escalado Re debido a la baja velocidad de agitación requerida $\mathrm{y}$ al incremento en la velocidad superficial del aire para tratar de mantener en ambas escalas el mismo flujo de aire por unidad de volumen del biorreactor (restricción para los dos criterios de escalado).

Las grandes diferencias en cuanto a los perfiles de aire utilizando el criterio P/V (Figura 2 c) se deben a que la velocidad requerida para mantener 


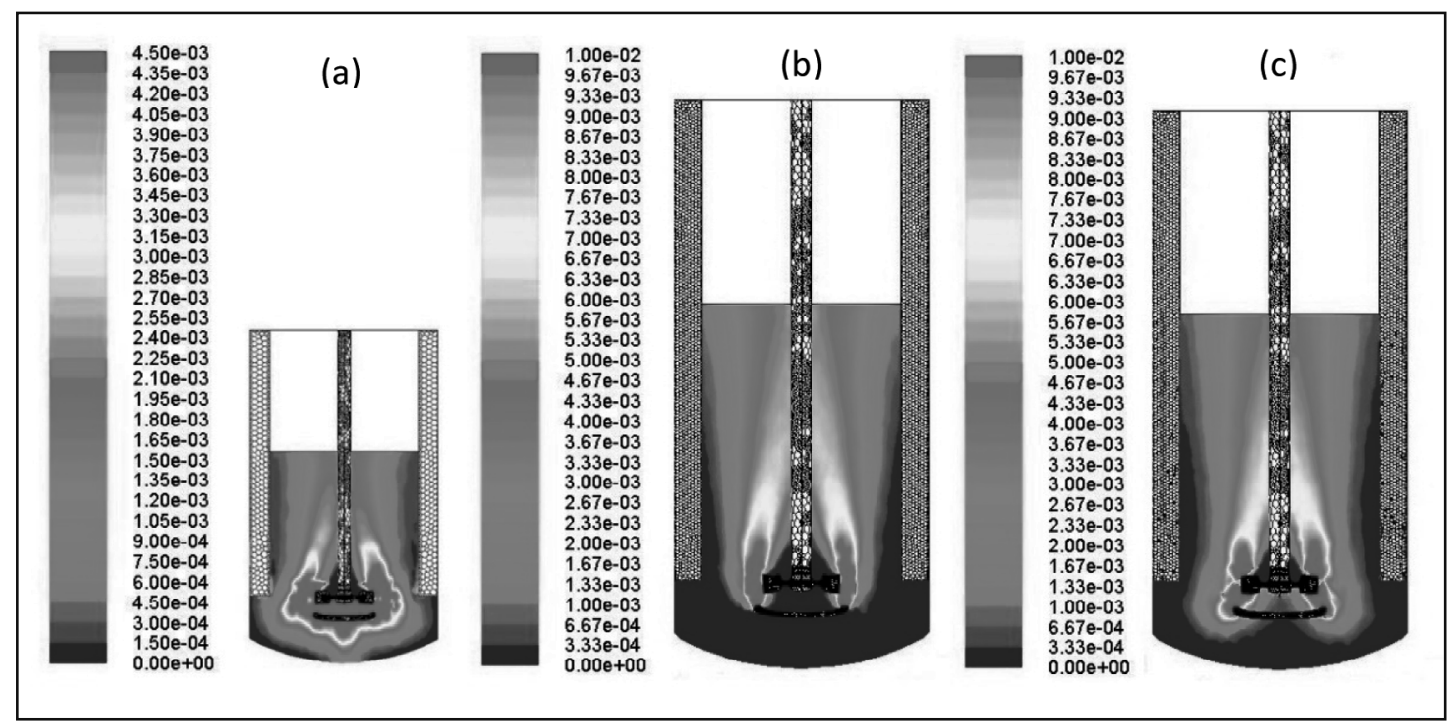

Figura 2. Fracción en volumen de la fase dispersa (aire) para los biorreactores de (a) 0,050 $\mathrm{m}^{3} \mathrm{y}$ $0,500 \mathrm{~m}^{3}$ aplicando los criterios de escalado (b) Re y (c) P/V.

este criterio constante en ambas escalas es mayor respecto del criterio Re; por lo tanto, las fuerzas centrífugas se incrementan, y el aire es capturado detrás de las aspas del agitador, fenómeno que disminuye el escape de las burbujas del biorreactor, lo que genera una mejor dispersión de las burbujas de aire y un ambiente hidrodinámico gobernado por fenómenos de ruptura de burbujas que trae como consecuencia una mejor transferencia de oxígeno (valores altos de $k_{L} a$ ).

La dispersión del gas se produce principalmente en las zonas cercanas a la turbina. Este es arrastrado a las zonas de menor presión estática existentes detrás de las aspas del agitador. El gas que sale del difusor junto con una gran parte de gas recirculado es arrastrado a estas cavidades.

En una fermentación aeróbica, el aire fluye de forma continua a través del reactor de tanque agitado. Es por tal razón que la distribución de tamaño de burbujas resulta de la interacción de mecanismos físicos como la ruptura y coalescencia. El parámetro más importante para analizar estos mecanismos hidrodinámicos es el diámetro medio de sauter. En las Figuras 3.1 y 3.2 se presentan los contornos del diámetro medio de sauter en los biorreactores de 0,050 (a) y $0,500 \mathrm{~m}^{3}$, aplicando los criterios de escalado (b) Re y (c) P/V. Detrás de las aspas se acumulan las burbujas para luego

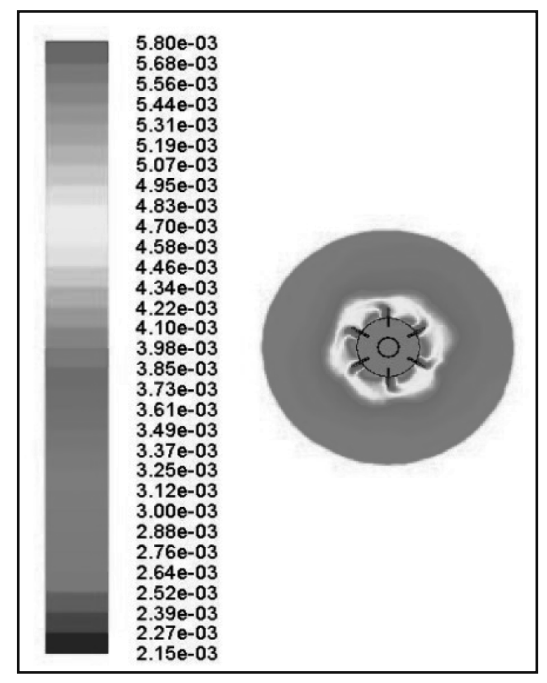

Figura 3.1. Diámetro medio de sauter $d_{32}(\mathrm{~m})$ de la fase dispersa (aire) del biorreactor $0,050 \mathrm{~m}^{3}$. Corte transversal a $0,12 \mathrm{~m}$ de altura.

ser dispersadas (Figura 3.1), lo que confirma el fenómeno de coalescencia en estas aéreas. Este comportamiento también ha sido reportado por Kerdouss [11].

Como se explicó anteriormente, el fenómeno de flotabilidad encontrado en el biorreactor de $0,500 \mathrm{~m}^{3}$, aplicando el criterio de escalado $\mathrm{Re}$ constante (Figura 3.2 b), influye negativamente 


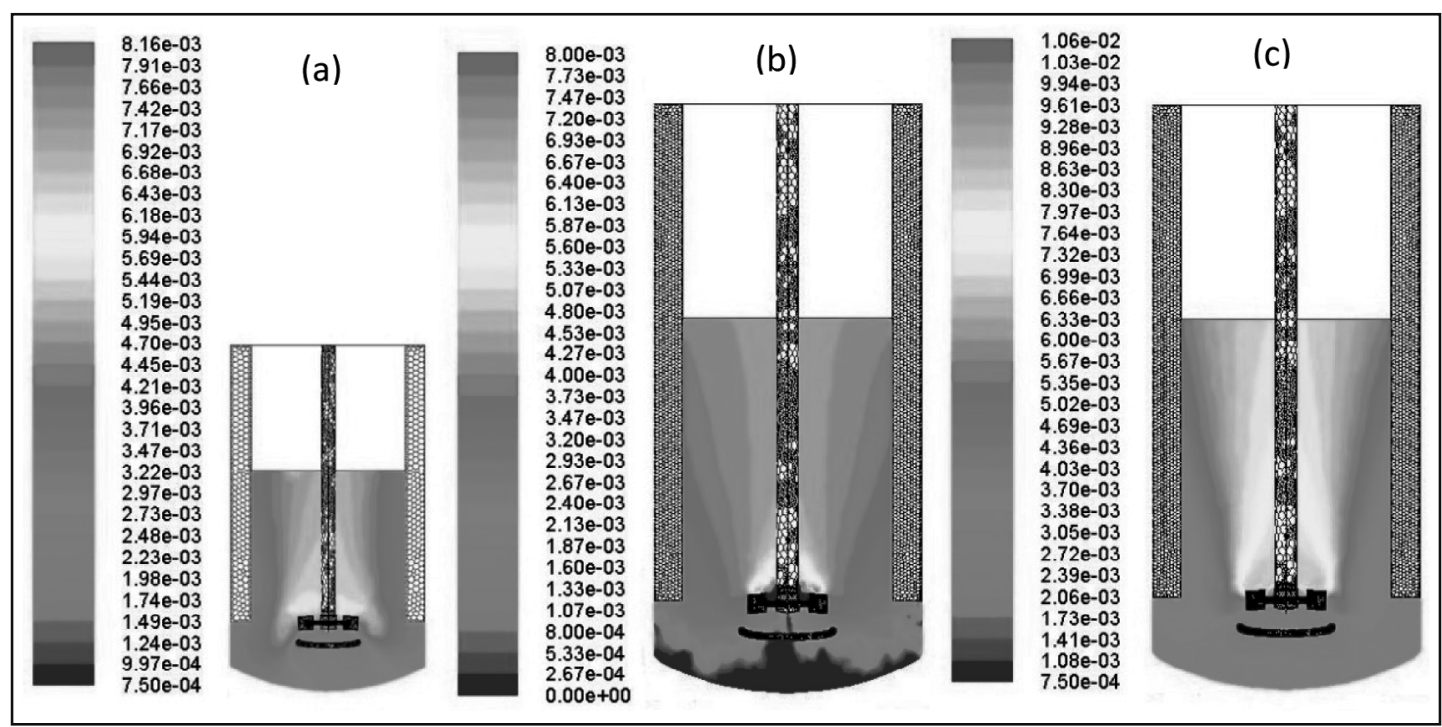

Figura 3.2. Diámetro medio de sauter $d_{32}(\mathrm{~m})$ de la fase dispersa (aire) para los biorreactores de (a) $0,050 \mathrm{~m}^{3}$ y $0,500 \mathrm{~m}^{3}$ aplicando los criterios de escalado (b) Re y (c) P/V.

en la dispersión de burbujas por lo que el aire permanece menor tiempo en el biorreactor y por esta razón la interacción entre las burbujas debida a los fenómenos de rompimiento, es débil, lo que genera zonas muertas en el fondo del biorreactor, donde las burbujas de aire no alcanzan a ser dispersadas. Por lo tanto estas zonas tienden a ser poco oxigenadas según los bajos valores de $k_{L} a$ simulados (Figura 6 b). Este fenómeno también ha sido reportado por Wang [4] en columnas de burbujeo a escala de laboratorio.

Con el incremento de escala, el fenómeno de coalescencia puede ser importante en un proceso líquido gaseoso. Tal es el caso que se presenta con el criterio de escalado P/V (Figura $3.2 \mathrm{c}$ ) donde el comportamiento no ideal desencadenado por la pérdida de homogeneidad debida al incremento de escala observado en el biorreactor de $0,500 \mathrm{~m}^{3}$ es causado por los fenómenos de ruptura y coalescencia, ya que en las zonas comprendidas entre el fondo del biorreactor, la turbina de agitación y los bafles deflectores, donde la disipación de la energía cinética turbulenta es más alta y los tamaños de remolinos son más bajos (Figura 5 c), el fenómeno de ruptura reduce significativamente el tamaño de las burbujas, causando de esta manera la deformación de las mismas hasta causar el rompimiento, como se observa gradualmente desde el fondo del biorreactor hasta la superficie a excepción del centro de rotación; por lo tanto, existe una mejor transferencia de oxígeno que en las zonas cercanas a la superficie del fluido líquido donde el fenómeno de coalescencia generado por las altas velocidades tangenciales, la baja disipación de la energía cinética turbulenta y el incremento en el tamaño de remolinos ocasionan comportamientos no ideales en el centro de agitación que deben evitarse durante un proceso de escalado mediante la instalación de una segunda turbina en esta zona del biorreactor.

En la Figura 4 se presentan los perfiles de velocidad del gas calculados por CFD en los biorreactores de (a) 0,050 y $0,500 \mathrm{~m}^{3}$, aplicando los criterios de escalado (b) Re y (c) P/V.

Se observan perfiles de velocidad desde $0,1 \mathrm{a} 1 \mathrm{~m} / \mathrm{s}$ para el caso del biorreactor a escala de $0,050 \mathrm{~m}^{3}$ (Figura 4 a) mientras que los perfiles encontrados para el caso del escalado con los criterios empíricos Re y P/V (Figuras 4 b y 4 c) los perfiles de velocidad del aire se reducen observándose intervalos desde 0 a 0,55 y desde 0,2 a $0,8 \mathrm{~m} / \mathrm{s}$.

El criterio $\mathrm{P} / \mathrm{V}$ presenta mayores similitudes con el biorreactor de $0,050 \mathrm{~m}^{3}$ en cuanto a los perfiles de velocidad de aire en el fondo del biorreactor y en las zonas cercanas a la turbina, en su comparación 


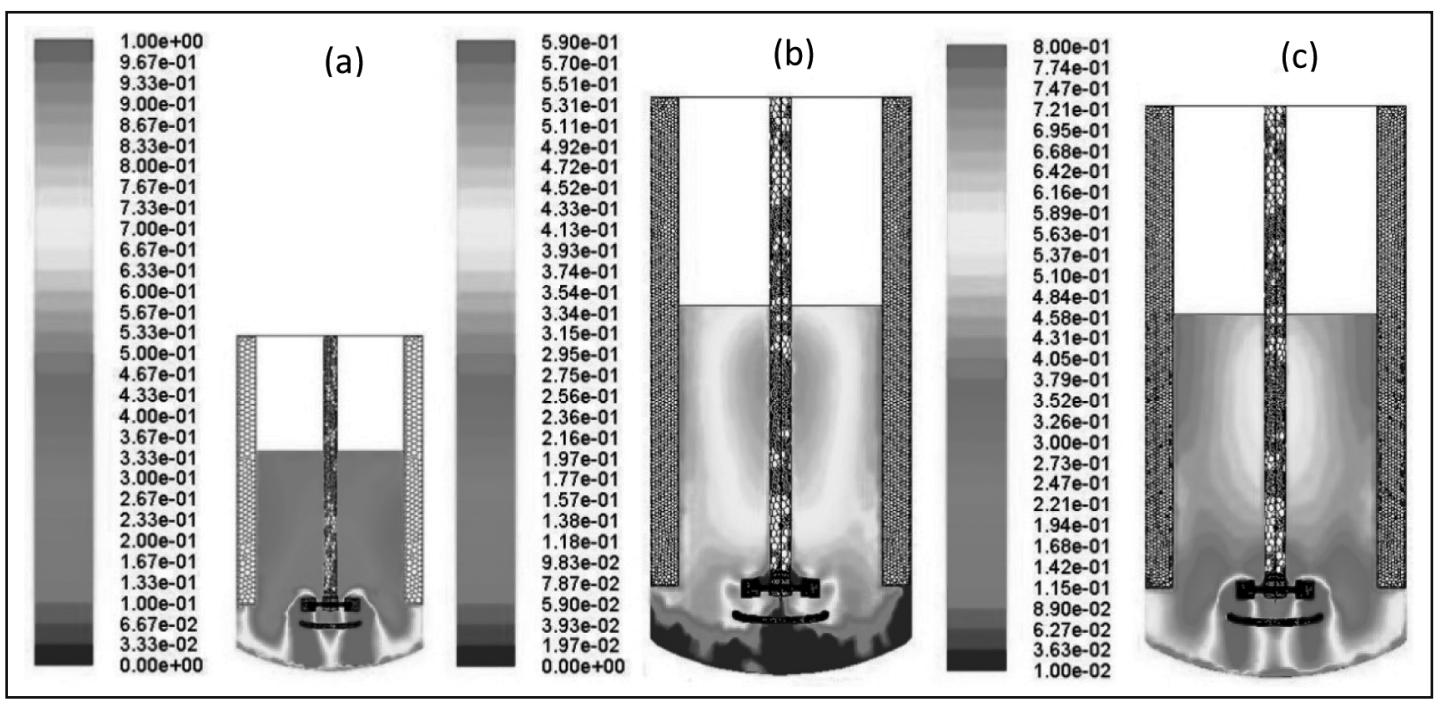

Figura 4. Perfiles de velocidad (m/s) de la fase dispersa (aire) para los biorreactores de (a) $0,050 \mathrm{~m}^{3} \mathrm{y}$ $0,500 \mathrm{~m}^{3}$ aplicando los criterios de escalado (b) Re y (c) P/V.

con los perfiles simulados con el criterio Re. Estos altos valores de velocidad encontrados en el fondo del biorreactor conducen a una mejor recirculación de aire y como consecuencia la transferencia de oxígeno $k_{L} a$ se incrementa en estas zonas (Figura $6 \mathrm{c}$ ). Sin embargo la pérdida de homogeneidad con el incremento de escala genera comportamientos no ideales en zonas alejadas de la turbina de agitación que se presentan en los dos criterios de escalado (Re y P/V).
Lo anterior, también es reflejado en la simulación del tamaño de remolinos mediante el diámetro de Kolmogórov (Figura 5) que muestra en todos los casos los valores más bajos en las zonas ubicadas entre la turbina y el difusor de aire y también en las paredes de los bafles deflectores. La ruptura de burbujas, como se explicó anteriormente, es analizada en términos de la interacción de las burbujas con los remolinos turbulentos. Estos remolinos turbulentos incrementan la energía de superficie de las burbujas hasta causar la deformación. Es por eso que en las

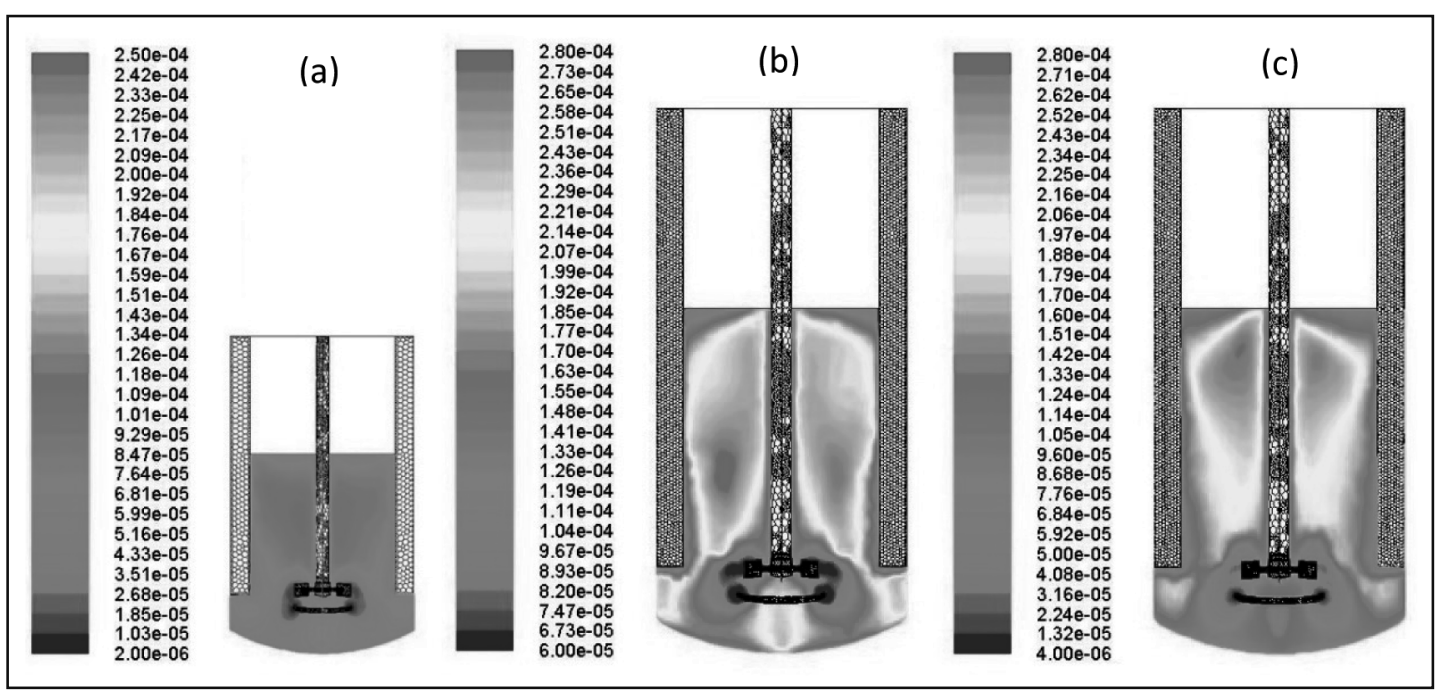

Figura 5. Perfiles del diámetro de Kolmogórov (m) para los biorreactores de (a) 0,050 m y 0,500 $\mathrm{m}^{3}$ aplicando los criterios de escalado (b) Re y (c) P/V. 


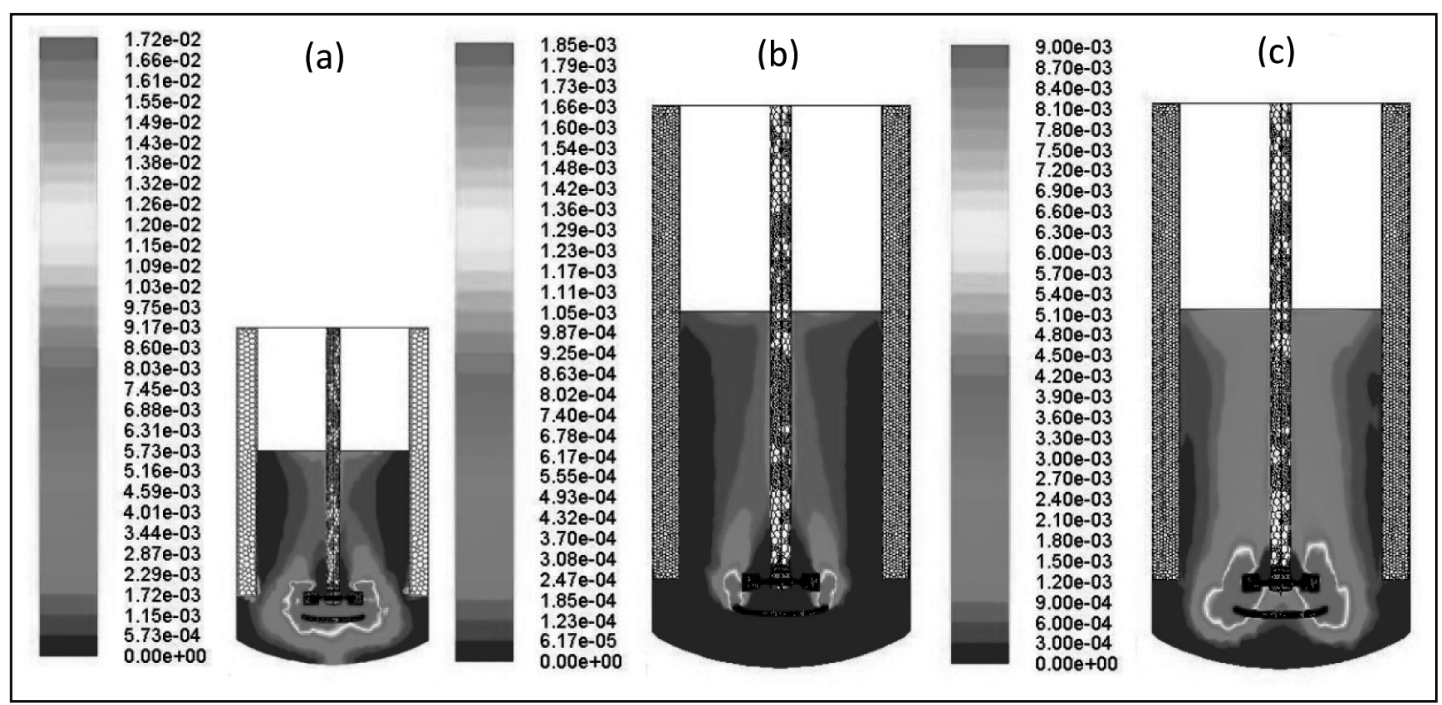

Figura 6. Perfiles del coeficiente de transferencia de masa $k_{L} a\left(\mathrm{~s}^{-1}\right)$ para los biorreactores de (a) $0,050 \mathrm{~m}^{3}$ y $0,500 \mathrm{~m}^{3}$ aplicando los criterios de escalado (b) Re y (c) P/V.

simulaciones se observa que la ruptura ocurre si el incremento en la energía de superficie, generada según el tamaño de remolinos, alcanza un valor crítico (Figuras 3.2 y 5). Por lo tanto, estas zonas son candidatas a una transferencia de masa aceptable como se observa en la Figura 6 para el crecimiento de microorganismos aerobios. Sin embargo, la teoría del daño celular debida a la turbulencia sugiere que si una célula es menor al diámetro de remolinos de Kolmogórov, la entidad biológica no sufrirá daño celular [45]. El tamaño promedio de algunas células animales se encuentra en el orden de 15-18 $\mu \mathrm{m}$ y teniendo en cuenta los valores mínimos de tamaño de remolinos simulados mediante $\mathrm{CFD}(4$ $\mu \mathrm{m})$ aplicando el criterio de escala $\mathrm{P} / \mathrm{V}$, se puede afirmar que se espera que ocurra daño microbiano en su estructura celular; por lo tanto, es necesario utilizar otro sistema de agitación cuando el objetivo es el cultivo de células animales.

El $k_{L} a$ es el parámetro que se usa comúnmente para describir la eficiencia de un sistema de aireación. Para calcular los valores de $k_{L} a$ a través de simulaciones en CFD se utilizó la teoría de la penetración de Higbies, en la cual el valor de $k_{L} a$ se obtiene como el producto del coeficiente de transferencia de masa de la fase líquida $k_{L}$ y el área interfaz $a$ [37]. El área interfaz se expresa como una función de la fracción local de volumen de gas y el diámetro de sauter local. Se observan similitudes entre los valores calculados por CFD a escala de $0,050 \mathrm{~m}^{3}$ y los datos obtenidos experimentalmente (Tabla 1 y Figura 6). Lo que significa que los términos de ruptura y coalescencia del modelo de balance poblacional predicen con buen acercamiento los fenómenos de transferencia de masa involucrados en las condiciones estudiadas a escala de $0,050 \mathrm{~m}^{3}$. Partiendo de esta información se simularon los valores de $k_{L} a$ para el biorreactor de $0,500 \mathrm{~m}^{3}$ con base en los criterios de escalado Re y P/V (Figura 6). Los resultados demuestran el efecto proporcional que ocurre en los valores de $k_{L} a$ con el incremento de la potencia consumida en cada criterio de escalado. Acontecimiento que ya ha sido verificado por investigaciones experimentales [39-41].

El aire en contacto con las aspas de la turbina tiende a disminuir el coeficiente de retardo o rozamiento asociado con el giro de la turbina y la consecuente disminución del consumo de potencia [42].

En la Tabla 1 se presentan los valores de potencia consumida con aireación $P_{g}$ y velocidad en la punta del impulsor $N D a$ calculados por CFD y comparados por las correlaciones mencionadas en el apartado de materiales y métodos para los biorreactores de 0,050 y $0,500 \mathrm{~m}^{3}$. Se observan similitudes entre los valores calculados por CFD (Tabla 1) y los datos obtenidos por las correlaciones. 
Tabla 1. Comparación de valores de CFD (NDa: velocidad en la punta del agitador; Pg: Potencia con aireación y $k_{L} a$ : Coeficiente de transferencia de masa) con datos obtenidos por correlaciones y técnicas experimentales (Exp).

\begin{tabular}{|c|c|c|c|c|c|c|}
\hline \multirow{2}{*}{ Criterio de Escalado } & \multicolumn{2}{|c|}{$\operatorname{NDa}(\mathbf{m} / \mathbf{s})$} & \multicolumn{2}{|c|}{$\operatorname{Pg}(\mathrm{W})$} & \multicolumn{2}{|c|}{$k_{L} a\left(\mathrm{~s}^{-1}\right)$} \\
\hline & Ec. (31) & CFD & Ec. (32) & CFD & Exp. & CFD \\
\hline $\begin{array}{l}\text { Referencia } \\
\left(0,050 \mathrm{~m}^{3}\right)\end{array}$ & 1,25 & 1,32 & 2,31 & 2,13 & 0,0190 & 0,0172 \\
\hline $\begin{array}{c}R e \\
\left(0,500 \mathrm{~m}^{3}\right)\end{array}$ & 0,63 & 0,65 & 1,5783 & 1,68 & \multicolumn{2}{|c|}{ 0,00185 (CFD) } \\
\hline $\begin{array}{c}P / V \\
\left(0,500 \mathrm{~m}^{3}\right)\end{array}$ & 1,63 & 1,70 & 20,90 & 20,69 & \multicolumn{2}{|c|}{ 0,0090 (CFD) } \\
\hline
\end{tabular}

Lo anterior argumenta que el modelo de balance poblacional acoplado a CFD predice con buen acercamiento los fenómenos de turbulencia en las condiciones hidrodinámicas estudiadas. En este trabajo se encontró que la transferencia de masa tiene una muy clara estructura definida por la hidrodinámica y los fenómenos de coalescencia y rompimiento de burbujas. El conocimiento acerca de la transferencia de masa en cultivos microbianos a gran escala es importante para determinar las zonas muertas poco oxigenadas que pueden afectar las actividades metabólicas de un cultivo celular, que se pueden presentar como consecuencia de un proceso de escalado.

Esta estrategia presentada en esta investigación de predicción de problemas que se pueden generar durante la etapa de escalado de un cultivo de microorganismos aerobios mediante CFD, puede ser una herramienta poderosa para comprender y solucionar problemas de transferencia líquidogaseosa que comúnmente ocurren en cultivos celulares. De esta manera, la información obtenida a partir de simulaciones en CFD de las heterogeneidades en cultivos de microorganismos aerobios podría ser usada para optimizar el escalado de un bioproceso.

\section{CONCLUSIONES}

El modelo de CFD aplicado en este trabajo simula los efectos hidrodinámicos generados por los criterios de escalado evaluados (Re y P/V) y predice tendencias hidrodinámicas que afectan un proceso de escalado. La velocidad en la punta del agitador, la potencia consumida con aireación y el coeficiente de transferencia de oxígeno calculados mediante CFD han sido verificados satisfactoriamente en correspondencia con datos experimentales obtenidos en esta investigación y correlaciones de la bibliografía. El criterio de escalado P/V utilizando una turbina Rushton presenta mejores similitudes hidrodinámicas que el criterio de escalado Re con referencia al biorreactor de $0,050 \mathrm{~m}^{3}$; sin embargo, el alto consumo de potencia y el posible daño microbiano generado por la turbulencia en cultivos de células animales son desventajas en prototipos a gran escala.

\section{REFERENCIAS}

[1] F. Bylund, E. Collet, S.O. Enfors and G. Larsson. "Substrate gradient formation in the large-scale bioreactor lowers cell yield and increases by-product formation". Bioprocess and Biosystems Engineering. Vol. 18, Issue 3, pp. 171-180. 1998. DOI: $10.1007 / \mathrm{s} 004490050427$.

[2] E. Dunlop. "Micromixing in fermentors: Metabolic changes in Saccharomyces cerevisiae and their relationship to fluid turbulence". Biotechnology and Bioengineering. Vol. 36 № 8, pp. 854-864. October 1990. DOI: 10.1002/bit.260360816.

[3] C.J. Hewitt, G.N. Caron, B. Axelsson, C.M. Mcfarlane and A.W. Nienow. "Studies related to the scale-up of high-cell-density E. coli fed-batch fermentations using multiparameter flow cytometry: Effect of a changing microenvironment with respect to glucose and dissolved oxygen concentration". Biotechnology and Bioengineering. Vol. 70 No 4, pp. 381-390. 2000. ISSN: 1097-0290.

[4] T. Wang, J. Wang and Y. Jin. "CFD-PBM Coupled Model for Gas-Liquid Flows". AIChE Journal. Vol. 52 No 1, pp. 125-140. 2006. DOI: 10.1002/aic.10611. 
[5] M. Jenne and M. Reuss. "A critical assessment on the use of k-e turbulence models for simulation of the turbulent liquid flow induced by Rushton turbine in baffled stirred-tank reactors". Chemical Engineering Science. Vol. $54 \mathrm{~N}^{\circ}$ 17, pp. 3921-3941. September 1999. DOI: 10.1016/S0009-2509(99)00093-7.

[6] J.Y. Luo, R.I. Issa and A.D. Gosman. "Prediction of impeller induced flows in mixing vessels using multiple frames of reference". In IchemE Symposium Series. Institution of Chemical Engineers. Vol. 136, pp. 549-556. 1994.

[7] G. Micale, A. Brucato and F. Grisafi. "Prediction of flow fields in a dual-impeller stirred tank". AICHE Journal. Vol. $45 \mathrm{~N}^{\circ} 3$, pp. 445-464. 1999.

[8] K. Rutherford, K.C. Lee, S.M.S. Mahmoudi and M. Yianneskis. "Hydrodynamic characteristics of dual rushton impeller stirred vessels. AICHE Journal". Vol. 42 $\mathrm{N}^{\circ}$ 2, pp. 332-346. 1996. DOI: 10.1002/ aic.690420204.

[9] A.D. Tabor, G. Gosman and R.I. Issa. "Numerical simulation of the flow in a mixing vessel stirred by a Rushton Turbine". IChemE Symposium Series. Vol. 140, pp. 25-34. 1996. DOI:10.1016/j.cep.2005.06.006.

[10] A. Bakker and H.E.A. Van den Akker. "A computational model for the gas-liquid flow in stirred reactors". Transactions of IChemE. Vol. $72 \mathrm{~N}^{\circ} \mathrm{A} 4$, pp. 594-606. July 1994. ISSN: 0888-5885.

[11] F. Kerdouss, A. Bannari, P. Proulx, R. Bannari, M. Skrga and Y. Labrecque."Twophase mass transfer coefficient prediction in stirred vessel with a CFD model". Computers and Chemical Engineering. Vol. $32 \mathrm{~N}^{\circ} 22$, pp. 1943-1955. August 2008. DOI:10.1016/j. compchemeng. 2007.10.01.

[12] F. Kerdouss, L. Kiss, P. Proulx, J.F. Bilodeau and C. Dupuis. "Mixing characteristics of an axial flow rotor: Experimental and numerical study". International Journal of Chemical Reactor Engineering. Vol. $3 \mathrm{~N}^{\circ}$ A35. 2005. ISSN: 1542-6580.

[13] F. Kerdouss, A. Bannari, P. Proulx, R. Bannari, M. Skrga and Y. Labrecque. "Two-phase mass transfer coefficient prediction in stirred vessel with a CFD model. Computers and Chemical Engineering". Vol. $3 \mathrm{~N}^{\circ}$ 8, pp. 1-13. 2007. ISSN: 0098-1354.
[14] G.L. Lane, M.P. Schwarz and G.M. Evans. "Numerical modeling of gas-liquid flow in stirred tank. Chemical Engineering Science". Vol. $60 \mathrm{~N}^{\circ} 8-9$, pp. 2203-2214. 2005. DOI: 10.1016/j.ces.2004.11.046.

[15] C.H. Venneker and H. Derksen Van Den Akker. "Population balance modeling of aerated stirred vessels based on CFD". AICHE Journal. Vol. 48 No 4, pp. 673-684. 2002. DOI: $10.1002 /$ aic.690480404.

[16] F. Scargiali, A. D'Orazio, F. Grisafi, A. Brucato."Modelling and Simulation of GasLiquid Hydrodynamics in Mechanically Stirred Tanks". Chemical Engineering Research and Design, Vol. $85 \mathrm{~N}^{\circ} 5$, pp. 637646. 2007. DOI: $10.1205 /$ cherd0624.

[17] G.R. Kasat, A.B. Pandit, y V.V. Ranade. "CFD Simulation of Gas-Liquid Flows in a Reactor Stirred by Dual Rushton Turbines". International Journal of Chemical Reactor Engineering. Vol. 6, pp. 1-28. 2008. ISSN: 1542-6580.

[18] Lu. Wei-Ming. "Multiple Impeller Gas-Liquid Contactors". Informe técnico. National Taiwan University. 2004. ISBN: 957-564-947-7.

[19] V.V. Ranade and J.B. Dommeti. "Computational Snapshot of flow generated by axial impellers in baffled stirred vessels". I Chem. Vol. 74 No 4, pp. 476-484. 1990. ISSN 0263-8762.

[20] V.V. Ranade. "Computational Flow Modeling for Chemical Reactor Engineering". Process Systems Engineering Series. Academic Press. 2002. ISBN: 0-12-576960-1.

[21] M. Jahoda, L. Tomášková, M. Moštěk. "CFD prediction of liquid homogenization in a gas-liquid stirred tank". Chemical Engineering Research and Design. Vol. 87 $\mathrm{N}^{\circ}$ 4, pp. 460-467. April 2009. DOI: 10.1016/j. cherd.2008.12.006.

[22] E.M. Marshall and A. Bakker, "Computational fluid mixing". Informe técnico. Fluent-Inc. 2002. ISBN: 0-9719532-0-1.

[23] M. Laakkonen, P. Moilanen, V. Alopaeus and J. Aittamaa. "Modelling Local Gas-Liquid Mass Transfer in Agitated Vessels". Chemical Engineering Research and Design. Vol. 85 $\mathrm{N}^{\circ}$ 5, pp. 665-675. 2007. DOI: 10.1205/ cherd0617

[24] R. Panneerselvam and S. Savithri. "Investigations on hydrodynamics and mass 
transfer in gas-liquid stirred reactor using computational fluid dynamics". Chemical Engineering Science. Vol. $66 \mathrm{~N}^{\circ} 14-15$, pp. 3108-3124. July 2011. DOI: $10.1016 /$ j. ces.2011.03.00.

[25] M. Martinov and S.D. Vlaev. "Increasing Gas-Liquid Mass Transfer in Stirred Power Law Fluids by Using a New Saving Energy Impeller". Chem. Biochem. Eng. Vol. 16 $\mathrm{N}^{\circ}$ 1, pp. 1-6. 2002. ISSN: 0352-9568.

[26] W. Tiefeng and W. Jinfu. "Numerical simulations of gas-liquid mass transfer in bubble columns with a CFD-PBM coupled model". Chemical Engineering Science. Vol. $62 \mathrm{~N}^{\circ}$ 24, pp. 7107-7118. December 2007.

[27] P. Chen, M.P. Dudukovic and J. Sanyal. "Numerical simulation of bubble columns flows: effect of different breakup and coalescence closures". Chemical Engineering Science. Vol. $60 \mathrm{~N}^{\circ}$ 4, pp. 1085-1101. 2005. ISSN 0009-2509.

[28] CRC Handbook. Handbook of Chemistry and Physics (83rd ed.). Boca Raton, FL, USA: CRC Press LLC. 2002.

[29] A. Junker. "Scale-Up Methodologies for Escherichia coli and Yeast Fermentation Processes". Journal of Bioscience and Bioengineering. Vol. $97 \mathrm{~N}^{\circ}$ 6, pp. 347-364. 2004. ISSN: 1347-4421.

[30] Ansys fluent v13. User's Manual. Fluent Inc. Centrera Resource Park, 10 Cavendish Court. Lebanon, USA. 2011.

[31] M. Ishii and N. Zuber. "Drag coefficient and relative velocity in bubbly, droplet or particulate flows". AIChE Journal. Vol. 25 $\mathrm{N}^{\circ}$ 5, pp. 843-855. June 1979. DOI: $10.1002 /$ aic.690250513.

[32] S.E. Elgobashiand M.A. Rizk. "A twoequation turbulence model for dispersed dilute confined two-phase flows". International Journal of Multiphase Flow. Vol. 15 $\mathrm{N}^{\circ} 1$, pp. 119-133. February 1989. DOI: 10.1016/0301-9322(89)90089-X

[33] M.J. Hounslow, R.L. Ryall and V.R. Marschall. "A Discretized Population Balance for Nucleation, Growth and Aggregation". AIChE Journal. Vol. $34 \mathrm{~N}^{\circ} 11$, pp. 18211832. November 1988. DOI: $10.1002 /$ aic.690341108.
[34] J.D. Litster, D.J. Smit and M.J. Hounslow. "Adjustable Discretization Population Balance for Growth and Aggregation". AIChE Journal. Vol. 41 No 3, pp. 591-603. 1995.DOI: 10.1002/aic.690410317.

[35] Ramkrishna, Population Balances: Theory and Applications to Particulate Systems in Engineering. Academic Press, San Diego, CA. 2000.ISBN: 0125769709. DOI: 10.1016/j. ces.2007.08.03.

[36] L. Hagesaether, H.A. Jakobsen, K. Hjarbo and H.F. Svendsen. "A coalescence and breakup module for implementation in CFD codes. In European Symposium on Computer Aided Process Engineering". Vol. 1, pp. 367-372. 2000.DOI:10.1016/S1570-7946(00)80063-2.

[37] J. Sanyal, D.L. Marchisio, R.O. Fox and K. Dhanasekharan. "On the comparison between population balance models for CFD simulation of bubble columns. Industrial Engineering and Chemical Research". Vol. 44, pp. 5063-5072. 2005. DOI: 10.1021/ ie049555j.

[38] Gambit 2. User's Manual to GAMBIT 2. Centrera Resource Park, 10 Cavendish Court. Fluent Inc. Lebanon, USA. 2004.

[39] F. Flórez, F. Pérez and M. de la Torre. "Scaleup of Bacillus thuringiensis fermentation based on oxygen transfer. Journal of Fermentation and Bioengineering". Vol. 83 $\mathrm{N}^{\circ}$ 6, pp. 561-564. 1997. DOI: 10.1016/ S0922-338X(97)81137-3.

[40] S. J. Arjunwadkar, K. Sarvanan, P. Kulkarni, and A.B. Pandit. "Gas-Liquid Mass Transfer in Dual Impeller Bioreactor". Biochem. Eng. Vol. $1 \mathrm{~N}^{\circ}$ 2, pp. 99-106. March 1998. ISSN: 1369-703X.

[41] V.B. Shukla, U Parasu, P.R. Kulkarni and A.B. Pandit. "Scale-up Biotransformation Process in Stirred Tank Reactor using Dual Impeller Bioreactor". Biochem. Eng. Vol. 8, pp. 19-29. 2001.DOI: 10.1016/ S1369-703X(00)00130-3.

[42] P.M. Doran. "Bioprocess Engineering Principles". Elsevier Science and Technology Books. University of New South Wales Sydney. Australia. 1995. ISBN: 978-0-12-220856-0.

[43] S. Nagata. "Mixing: Principles and Application". New York, NY. John Wiley \& Sons, pp. 59-62. 1975. 
Gelves, Benavides y Quintero: Predicción del comportamiento hidrodinámico en el escalado de un reactor de tanque agitado...

[44] K.R. Westerterp, L.L. van Dierendonck and J.A. Kraa. "Interfacial areas in agitated gas-liquid contactors". Chem. Eng. Sci. Vol. 18, pp. 157-176. 1963. DOI:10.1016/0009-2509(63)85002-2.
[45] V.V. Ranade. "Computational Flow Modeling for Chemical Reactor Engineering". Process Systems Engineering Series. Academic Press. 2002. 